\title{
Nuevo registro para Avellanita bustillosii Phil. (Euphorbiaceae) en la Región de Valparaíso, Chile
}

\author{
New record for Avellanita bustillosii Phil. (Euphorbiaceae) in the Region of Valparaíso, Chile
}

\author{
Arón Cádiz-Véliz ${ }^{1,3 *}$ \& Carolina Aliaga-Reyes ${ }^{2}$ \\ ${ }^{1}$ Instituto de Biología, Facultad de Ciencias, Pontificia Universidad Católica de Valparaíso, Campus Curauma, Avenida Universidad 330, Valparaíso, Chile. \\ ${ }^{2}$ Instituto de Geografía, Facultad de Historia, Geografía y Ciencia Política, Pontificia Universidad Católica de Chile, Campus San Joaquín, Avenida Vicuña Mackenna 4860, \\ Santiago, Chile. \\ 3Jardín Botánico Nacional, Camino El Olivar 305 El Salto, Viña del Mar, Chile. \\ *Email: aron.cadiz.veliz@gmail.com
}

\section{ABSTRACT}

Two new populations of Avellanita bustillosii Phil., an endemic endangered (EN) shrub of Chile central, is documented near Putaendo, Valparaíso. This new record constitutes the fifth and sixth population of the species, which extends the northern boundary of distribution and a establishes a new altitudinal boundary.

Avellanita bustillosii Phil. (Euphorbiaceae) es la única especie del género Avellanita, arbusto endémico del bosque esclerófilo de Chile Central (Villagrán \& Hinojosa 1997; Serra et al. 2005; Muñoz-Schick et al. 2012). Esta especie es catalogada como En Peligro (EN B1ab(iii)+2ab(ii)) de acuerdo con el Reglamento para la Clasificación de Especies Silvestres (RCE) según estado de conservación del Ministerio del Medio Ambiente en el primer proceso de clasificación, DS 151/2007 MINSEGPRES (Benoit 1989; Serra et al. 2005) y propuesta como en Peligro Crítico (CR) por Medina y Rojas (2018) sobre la base de criterios UICN. También es considerada como una de las especies leñosas más escasas de Chile (Serra et al. 2005) debido a su distribución restringida, población reducida y baja frecuencia en estado natural (Ministerio del Medio Ambiente 2007). La especie fue descrita por R.A. Phillippi (Phillippi 1864) en 1864 a partir de ejemplares recolectados por Vicente Bustillos en la Provincia de Colchagua, Región del Libertador Bernardo O'Higgins. Lamentablemente esta población no se ha vuelto a registrar y el ejemplar no incluye georreferencia (Serra et al. 2005).

Su distribución histórica abarca desde la Región de Valparaíso a la Región del Libertador Bernardo O'Higgins, comprendiendo un rango altitudinal entre los 350 a $880 \mathrm{~m}$ s.n.m. (Hechenleitner et al. 2005; Serra et al. 2005; Medina $\&$ Rojas 2018). Se han descrito cuatro poblaciones para la especie: en las cercanías de la Laguna de Aculeo en la Región Metropolitana (3350' S; 7056' O), siendo esta la más estudiada (Serra et al. 2005). Una segunda población fue descubierta por los naturalistas Jaffuel y Pirion (1921)

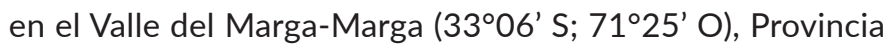
de Marga-Marga, Región de Valparaíso, esta población no se ha vuelto a encontrar desde su registro en 1910 (Ricci \& Benoit 2008). En 2004 se encontró una nueva población en la localidad de Lo Chancón (3460’ S; 7051' O), Provincia de Cachapoal, Región del Libertador Bernardo O’Higgins (Serra et al. 2005). En el año 2013 una última población fue descrita cerca del tranque Cauquenes, Provincia de Cachapoal (34¹6' S; 7041' O), Región del Libertador Bernardo O'Higgins, ampliando su rango de distribución hacia el sur (Medina \& Rojas 2018).

En este estudio se describen dos nuevas poblaciones para A. bustillosii, ampliando su límite de distribución hacia el norte y aumentando su rango altitudinal en la Quebrada de Granallas (32³6'43" S; 7046'33" O) y Quebrada Herrera (32³9'0.9" S; 7045’43" O), Provincia de San Felipe, Región de Valparaíso, en el cordón transversal de Altos de Putaendo, asociado al tipo forestal bosque esclerófilo.

MATERIALES REVISADOS

CHILE: Región de Valparaíso, Prov. de Valparaíso, Marga- 
Marga, 150 m, IX-1910. Jaffuel 46403 (CONC). Región de Valparaíso, Prov. de San Felipe, Putaendo, Granallas, 1298 m, IX-2016. Cádiz-Véliz A. 167868 (SGO). Región de Valparaíso, Prov. de San Felipe, Putaendo, Quebrada Herrera, 847 m, VIII2017. Cádiz-Véliz A. 169439 (SGO). Región Metropolitana, Prov. de Maipo, Laguna de Aculeo, 350 m, XI-1987. Macaya 136409 (CONC). Región Metropolitana, Prov. de Maipo, cerro Cantillana, 390 m, IX-2003. Marticorena A. 165576 (CONC). Región Metropolitana, Prov. de Maipo, Laguna de Aculeo, 363 m, XI-2004. Baxter et al. 176615 (CONC). Región Metropolitana, Prov. de Maipo. Laguna de Aculeo, 350 m, IX-1970. Kausel E. 109226 (SGO). Región del Libertador
Bernardo O’Higgins, Prov. de Cachapoal, Rancagua, GultroColihue, 708 m, XI-2014. Rojas G. 182676 (CONC). Región del Libertador Bernardo O’Higgins, Prov. de Cachapoal, Rancagua, Gultro-Colihue-Cauquenes, 905 m, V-2013.Rojas G. 166378 (SGO). Región del Libertador Bernardo O'Higgins, Prov. de Cachapoal, Rancagua, Tranque Cauquenes, $826 \mathrm{~m}$, XI-2013. Medina P. 166380 (SGO). Región del Libertador Bernardo O’Higgins, Prov. de Cachapoal, Lo Chancón, 520 m, XI-2004, MT. Serra, M. Ricci \& F. Luebert 151596 (SGO). (3406'S, 7051'O). Región del Libertador Bernardo O’Higgins, Prov. de Cachapoal, Lo Chancón, 477 m, V-2012, P. Medina 161145 (SGO).

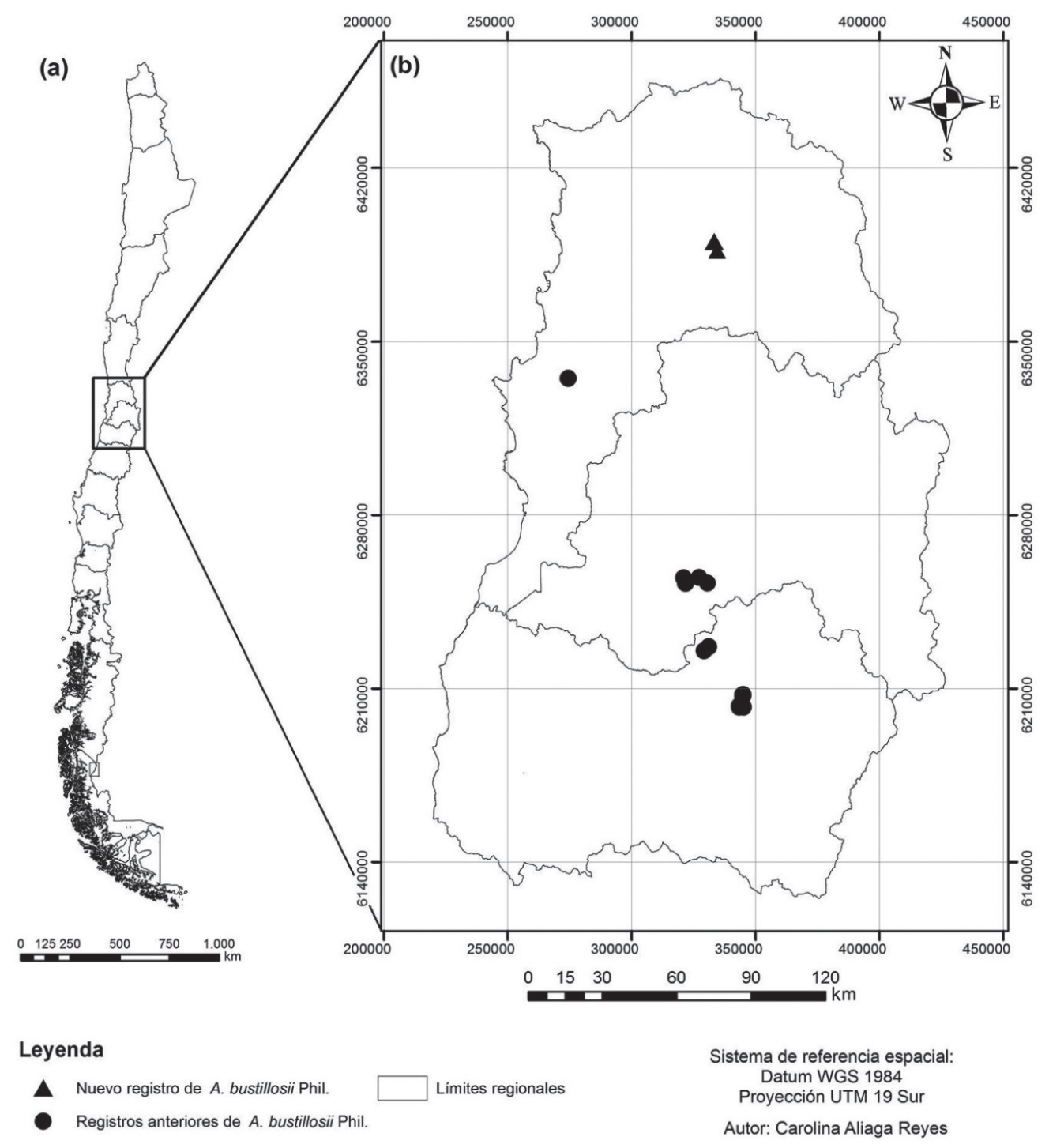

FIgURA 1. Mapa geográfico de distribución de Avellanita bustillosii Phil. A) Mapa de Chile continental. B) Regiones estudiadas. / Geographic distribution map of Avellanita bustillosii Phil. A) Map of continental Chile. B) Studied regions. 


\section{NueVo REgISTRo}

En el mes de noviembre de 2016, bajo una expedición para el estudio florístico del cordón de Altos de Putaendo, en la Quebrada de Granallas a una altitud de 1300 m.s.n.m. (Fig. 1), se descubrieron individuos de $A$. bustillosii creciendo al costado del sendero en un fondo de quebrada con abundante vegetación (Fig. 2a). Se identificó el piso vegetacional del área como "bosque mediterráneo andino de Quillaja saponaria Molina y Lithrea caustica Hook. \& Arn." (Luebert \& Pliscoff 2006) (Fig. 2b). La mayoría de los individuos se encontraban formando parte del sotobosque a escasos metros del cauce.

Se registró una población con sólo 17 individuos con tamaños que variaron entre 0,07 a 2,23 m de altura. Sólo dos individuos se encontraban con frutos y cinco con signos de herbivoría. A partir de este hallazgo, se procedió a registrar las localidades aledañas en busca de más individuos de A. bustillosii, encontrándose una segunda población en la localidad de Quebrada Herrera (Fig. 1), 3,2 km al sur de la quebrada de Granallas, en un rango altitudinal que varió entre 847 y los 1.660 m, entre el piso vegetacional "matorral arborescente esclerófilo mediterráneo interior de Quillaja Saponaria y Porlieria chilensis" y el "bosque esclerófilo mediterráneo andino de Kageneckia angustifolia y Guindilia trinervis" (Luebert \& Pliscoff 2006). Se registraron cerca de 100 individuos con tamaños que variaron entre los 0,30 a $1,50 \mathrm{~m}$, ninguno con frutos y la mayoría con signos de herbivoría. Se recolectó el material de referencia y se depositó en los herbarios SGO, JBN y VALPL. Se elaboró cartografía de distribución geográfica de la especie a partir de datos obtenidos de los herbarios SGO y CONC.

Según este registro, el nuevo límite septentrional de la especie se amplía al menos en $138 \mathrm{~km}$ lineales desde el anterior límite norte de distribución en la Laguna de Aculeo, puesto que la población de Marga-Marga no se ha vuelto a reportar, y su rango de distribución altitudinal se amplía en $780 \mathrm{~m}$.

El tipo de vegetación en el que participa $A$. bustillosii corresponde típicamente a formaciones boscosas de la Cordillera de la Costa dominadas por Cryptocarya alba (Molina) Looser y Peumus boldus Molina (Hechenleitner et al. 2005). Sin embargo, la población reportada en este estudio difiere en lo que refiere a las especies acompañantes, siendo dominante Quillaja saponaria y Lithrea caustica, sin presencia de $C$. alba ni $P$. boldus. Además se encuentra en la zona de estudio Kageneckia oblonga Ruiz \& Pav., Azara petiolaris (D. Don) I.M. Johnst., Colliguaja odorifera Molina y Escallonia illinita $\mathrm{C}$. Presl en sitios más húmedos.

Los individuos que presentaron signos de herbivoría se encontraron en sitios abiertos de fácil acceso para el ganado. Se ha señalado que $A$. bustillosii posee semillas comestibles (Pardo \& Pizarro 2013), hojas palatables por el ganado y es muy susceptible a las modificaciones de su hábitat (Hechenleitner et al. 2005). Aunque la zona del valle de Putaendo ha sido

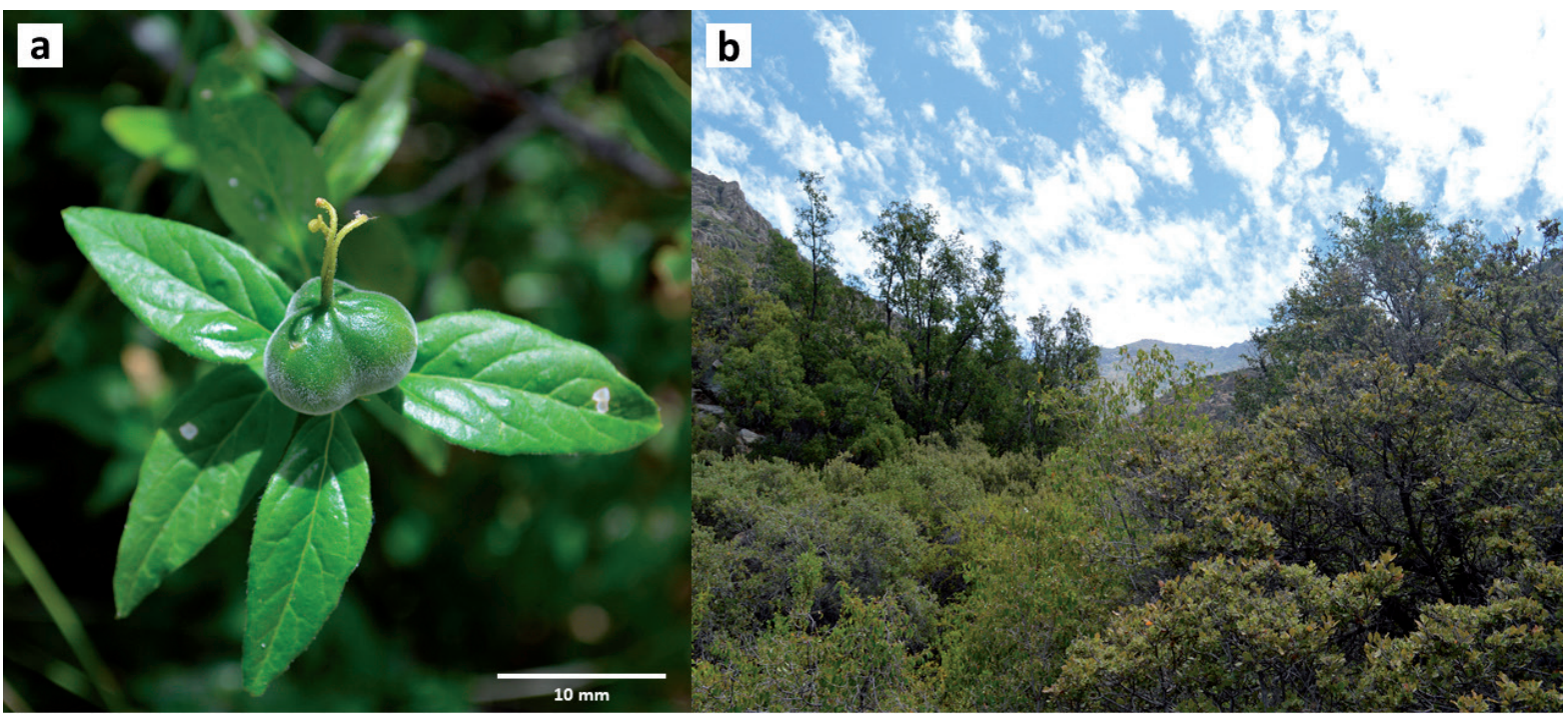

Fıgura 2. a) Individuo de Avellanita bustillosii Phil., observado en la Quebrada Granallas; detalle del fruto. b) Hábitat de A. bustillosii, bosque esclerófilo denso dominado por Quillaja saponaria y Lithrea caustica (Fotografías: A. Cádiz-Véliz). / a) Detail of Avellanita bustillosii Phil. fruit observed at Quebrada Granallas. b) Habitat of A. bustillosii, sclerophyllous forest dominated by Quillaja saponaria and Lithrea caustica (Photographs: A. Cádiz-Véliz). 
intensamente explotada desde el año 1600 por la extracción de leña, producción de carbón e intensa actividad ganadera, ejerciéndose una fuerte presión sobre la vegetación (Razeto et al. 2008), las actividades ganaderas en el área han ido disminuyendo en la actualidad como efecto de la reducción de la tenencia de ganado y su exclusión dentro de las áreas silvestres (autores, observación personal), lo que podría permitir la regeneración de estas poblaciones; sin embargo, existen nuevos proyectos mineros, actividad agroindustrial y sequía que amenazan esta zona. Debido a esto, es de suma importancia tomar medidas para la protección de la población in situ ya que sus poblaciones naturales no se encuentran protegidas dentro del Sistema Nacional de Áreas Silvestres Protegidas del Estado. Este hallazgo constituye un hito para $A$. bustillosii, ya que hace más de 100 años que no se registraba para la Región de Valparaíso.

\section{AGRADECIMIENTOS}

Se agradece a Gloria Rojas y Alicia Marticorena por el acceso al material de consulta y envío de información, y a Gastón Carvallo por las sugerencias al manuscrito.

\section{REFERENCIAS}

Benoit, I. 1989. Libro Rojo de la Flora Terrestre de Chile (Primera Parte). CONAF, Santiago. 157 pp.

Hechenleitner, P., Gardner, M., Thomas, P., Echeverría, C., Escobar, B., Bronwless, P., Martínez, C. 2005. Plantas Amenazadas del Centro-Sur de Chile. Distribución, Conservación y Propagación. Universidad Austral de Chile y Real Jardín Botánico de Edimburgo. 188 pp.

Jaffuel, F., Pirion, A. 1921. Plantas Fanerógamas del Valle de
Marga-Marga. Revista Chilena de Historia Natural 25: 350-405.

Luebert, F., Pliscoff, P. 2006. Sinopsis bioclimática y vegetacional de Chile. Editorial Universitaria, Santiago. 316 pp.

Medina, P., Rojas, G. 2018. Nueva localidad para Avellanita bustillosii Phil. (Euphorbiaceae) en la Región de O'Higgins y su estado de conservación. Gayana Botánica 75(1): 539-543.

Ministerio del Medio Ambiente. 2014. Quinto Informe Nacional de Biodiversidad de Chile ante el Convenio sobre la Diversidad Biológica (CBD). Ministerio del Medio Ambiente, Santiago. 140 pp.

Muñoz-Schick, M., Moreira-Muñoz, A., Moreira E. 2012. Origen del nombre de los géneros de plantas vasculares nativas de Chile y su representatividad en Chile y el mundo. Gayana Botánica 69(2): 309-359.

Pardo, O., Pizarro, J. 2013. Chile: Plantas alimentarias prehispánicas. Editorial Parina, Arica. 431 pp.

Philippi, R. A. 1864. Plantarum novarum chilensium. Centuriae inclusis quibusdam Mendocinis et Patagonicis. Linnaea 33: 1-308.

Razeto, J., Madrid, A., Del Campo, P., Cerda, J.C., Escobar, J., Carrasco, J., Pavlovic, D., Mella, J., Ortega, D. 2008. Santuario de la Naturaleza Serranía del Ciprés. Ediciones Almendral, San Felipe. 139 pp.

Ricci, M., Benoit, I. (eds.). 2008. Plan Nacional de Conservación de la avellanita, Avellanita bustillosii Phil. en Chile. CONAF, Santiago. 39 pp.

Serra, M., Luebert, F., Ricci, M. 2005. Nuevo registro para Avellanita bustillosii Phil. (Euphorbiaceae). Noticiario Mensual del Museo Nacional de Historia Natural 355: 11-15.

Villagrán, C., Hinojosa, L.F. 1997. Historia de los bosques del sur de Sudamérica, II: Análisis fitogeográfico. Revista Chilena de Historia Natural 70 (2): 1-267.

Received: 02.07.2018

Accepted: 24.04.2019 